\title{
Favorable outcome of haploidentical hematopoietic stem cell transplantation in Philadelphia chromosome-positive acute lymphoblastic leukemia: a multicenter study in Southwest China
}

Li Gao', Cheng Zhang ${ }^{1}$, Lei Gao ${ }^{1}$, Yao Liu', Yi Su², Sanbin Wang ${ }^{3}$, Bin Li ${ }^{4}$, Tonghua Yang ${ }^{5}$, Zhong Yuan ${ }^{6}$ and Xi Zhang ${ }^{1 *}$

\begin{abstract}
Background: Since the introduction of tyrosine kinase inhibitors (TKIs) into combination chemotherapy regimens, the majority of newly diagnosed Philadelphia chromosome-positive acute lymphoblastic leukemia (Ph+ ALL) patients have achieved complete remission (CR). However, without allogeneic hematopoietic stem cell transplantation (HSCT), long-term outcomes in adults remain unsatisfactory. Indeed, haploidentical HSCT has become a common treatment for adult patients who lack an HLA-matched donor, though limited data are available on the efficacy of haploidentical HSCT in Ph+ ALL patients.
\end{abstract}

Methods: We analyzed the clinical outcomes of $82 \mathrm{Ph}+\mathrm{ALL}$ patients who underwent haploidentical HSCT $(n=47)$ or HLA-matched HSCT $(n=35)$. Real-time quantitative reverse transcription polymerase chain reaction (qRT-PCR) was performed to assess BCR-ABL expression. All of the patients were treated with an imatinib-based regimen before undergoing HSCT. Imatinib treatment was resumed in the patients' posttransplantation following detection of BCR-ABL transcripts.

Results: All of the patients achieved neutrophil and platelet engraftment, with the exception of five patients who died prior to engraftment. Haploidentical HSCT was associated with higher incidences of acute graft-versus-host disease (GVHD) (51.1 vs. $25.7 \%, p<0.05)$ and chronic GVHD (48.9 vs. $25.7 \%, p<0.05)$ compared with HLA-matched HSCT, but there was no difference in the incidence of either grades III-IV acute GVHD or extensive chronic GVHD. The incidence of cytomegalovirus (CMV) infection was significantly higher in the patients treated with haploidentical HSCT than in those treated with HLA-matched HSCT (38.3 vs. $14.3 \%, p<0.05$ ). Haploidentical HSCT was associated with a significantly lower relapse rate compared with HLA-matched HSCT (44.8 vs. $19.1 \%, p<0.05$ ). There were no differences in non-relapse mortality (NRM), leukemia-free survival (LFS), or overall survival (OS) between the patients who received HLA-matched HSCT and those who underwent haploidentical HSCT.

Conclusions: Our data indicate that the incidence of NRM after HSCT is similar between the patients who receive HLA-matched donor cells and those who receive haploidentical donor cells and that haploidentical HSCT reduces the relapse rate. Haploidentical HSCT represents an encouraging treatment option for Ph+ ALL patients who lack a suitable HLA-matched donor.

\footnotetext{
* Correspondence: zhangxxi@sina.com

${ }^{1}$ Department of Hematology, Xinqiao Hospital, Third Military Medical University, Xinqiao Street, Shangpinba District, Chongqing 400037, China Full list of author information is available at the end of the article
} 


\section{Introduction}

Approximately $25-30 \%$ of adults and $3 \%$ of children with acute lymphoblastic leukemia (ALL) express the oncogenic BCR-ABL protein, which results from a 9;22 chromosomal translocation known as the Philadelphia $(\mathrm{Ph})$ chromosome [1]. Ph-positive ALL (Ph+ ALL) is a high-risk subset of ALL that is associated with a lower probability of complete remission (CR) than Ph-negative ALL (Ph- ALL) and that has an extremely poor prognosis [2]. Most studies have found that Ph+ ALL patients have a median survival time of 6-12 months [3, 4]. Although the introduction of tyrosine kinase inhibitors (TKIs) into the standard combination chemotherapy regimens for newly diagnosed Ph+ ALL allows over $95 \%$ of patients to achieve CR [5, 6], Ph+ ALL remains an unfavorable prognostic subgroup with an unacceptably high relapse rate. In the UKALLXII/ECOG2993 trial, the addition of imatinib to the chemotherapeutic cocktail in the absence of myeloablative allogeneic hematopoietic stem cell transplantation (allo-HSCT) did not result in a significant survival benefit [7]. Allo-HSCT during the first CR (CR1) remains the optimal curative treatment for Ph+ ALL to date [3]. The most therapeutically favorable treatment for Ph+ ALL is allo-HSCT from an HLA-identical sibling donor, but approximately $70 \%$ of patients lack a suitable sibling donor [8]. In China, matched unrelated donors [9] are found for only $20 \%$ of patients who lack a suitable sibling donor, and umbilical cord blood units are not always suitable for adult transplantation because they contain insufficient numbers of cells. However, nearly all patients have at least one HLA-haploidentical matched family member. Recent comparative studies have shown that acute leukemia patients who receive either haplotransplantation or HLAmatched transplantation have similar outcomes [10, 11], but the efficacy and safety of haplotransplantation in $\mathrm{Ph}+$ ALL patients have not been examined. Our current study compares the outcomes of patients who have been diagnosed with Ph+ ALL and treated with HSCT using cells from either haploidentical donors or matched donors.

\section{Materials and methods \\ Patients}

We analyzed the clinical data of 82 patients with $\mathrm{Ph}+\mathrm{ALL}$ who underwent either HLA-matched HSCT or haploidentical HSCT at one of five hospitals in southwest China between July 2007 and April 2011. The diagnosis of Ph+ ALL was based on the diagnostic criteria of the World Health Organization (WHO). The BCR-ABL fusion gene was detected by real-time quantitative polymerase chain reaction (PCR). The study was reviewed and approved by the ethics committees of the participating institutions. All of the patients included in this study provided informed consent in accordance with the Declaration of Helsinki.

\section{Conditioning regimen and imatinib therapy}

The patients undergoing HLA-matched HSCT were conditioned with 9.0-10.5 Gy total-body irradiation (TBI) on days -5 and -4 and $60 \mathrm{mg} / \mathrm{kg} /$ day intravenous cyclophosphamide (CY) on days -3 and -2 . The patients undergoing haploidentical HSCT were conditioned with 9.0-10.5 Gy TBI on days -7 and $-6,6 \mathrm{~g} / \mathrm{m}^{2} /$ day intravenous arabinosylcytosine (Ara-C) on days -5 to $-3,45 \mathrm{mg} / \mathrm{kg} /$ day intravenous $\mathrm{CY}$ on days -3 to -2 , and $2.5 \mathrm{mg} / \mathrm{kg} /$ day intravenous anti-thymocyte globulin (ATG) (Sanofi, SangStat, Lyon, France) on days -5 to -2 .

All of the patients received imatinib prior to transplantation. Imatinib was administered posttransplantation only if BCR-ABL transcripts were detectable by real-time quantitative PCR and if the patients could tolerate oral imatinib without developing gut graft-versushost disease (GVHD) or life-threatening infection.

\section{Donors, stem cell mobilization, and stem cell collection}

Peripheral blood (PB) and bone marrow (BM) cells were collected from the donors using standard mobilization protocols. Granulocyte colony stimulating factor (G-CSF) (5 $\mathrm{\mu g} / \mathrm{kg} /$ day; Filgrastim, Kirin Pharma Co., Ltd., Japan) was administered to the donors for 5-6 days to mobilize stem cells in the BM (G-BM) and PB (G-PB). Starting on the fifth day of G-CSF administration (day 1), G-PB cells were harvested by large-volume leukapheresis using a Fenwal CS3000 apparatus (Fenwal, Deerfield, IL, USA). If the number of mononuclear cells (MNCs) or CD34+ cells harvested was not sufficient for HLA-matched HSCT, additional G-PB cells were harvested on day 2. On day 2, G-BM cells were harvested for haploidentical HSCT. The goal was to collect at least $4 \times 10^{8} \mathrm{MNCs}$ and $2 \times 10^{6}$ CD34+ cells per kilogram of the recipient's body weight.

\section{GVHD prophylaxis and management}

The patients who received HLA-matched HSCT were treated posttransplantation with mycophenolate mofetil (MMF), cyclosporin A (CsA), and methotrexate (MTX) [12]. A total of $500 \mathrm{mg}$ (15 mg/kg for pediatric patients) of MMF (Roche Pharmaceutical, Ltd., Switzerland) were administered orally every $12 \mathrm{~h}$ from day 1 until day 30 . Continuous intravenous CsA (2.5 mg/kg/day) was administered starting on day -1 and was continued until the patient was able to tolerate oral medication. Next, $2.5 \mathrm{mg} / \mathrm{kg}$ CsA was administered orally twice per day to achieve a blood concentration of $200-300 \mathrm{ng} / \mathrm{mL}$ for 90 days. The dose of CsA was then gradually reduced. In total, $15 \mathrm{mg} / \mathrm{m}^{2}$ MTX was administered intravenously on day 1 , and $10 \mathrm{mg} / \mathrm{m}^{2}$ MTX was administered on days 3 and 6 . The patients who received haploidentical HSCT were treated posttransplantation with ATG, MMF, CsA, and MTX. In addition to the standard treatment with ATG, MMF was administered from day -7 until day 90. 
Continuous intravenous CsA $(1.5 \mathrm{mg} / \mathrm{kg} /$ day $)$ was started on day -7 , and the dose was increased to $2.5 \mathrm{mg} / \mathrm{kg} /$ day on day -1 , and it was replaced with oral medication. Over the next 150-180 days, the dose of CsA was gradually reduced. A total of $15 \mathrm{mg} / \mathrm{m}^{2}$ MTX were administered on day 1 , and $10 \mathrm{mg} / \mathrm{m}^{2}$ MTX were administered on days 3, 6 , and 11 .

The patients who developed GVHD were treated with $1-2 \mathrm{mg} / \mathrm{kg} /$ day methylprednisolone or prednisolone and $2.5 \mathrm{mg} / \mathrm{kg} /$ day CsA. The patients who developed steroidrefractory GVHD were treated with second-line immunosuppressive therapies, such as MMF, tacrolimus (FK506, Astellas, Japan), or anti-CD25 monoclonal antibodies (Novartis Pharma, Ltd., Switzerland).

\section{Evaluation and definitions}

The degree of hematopoietic chimerism was determined using a PCR-based assay that detects short tandem repeats in DNA. Full donor chimerism was defined by the detection of $95 \%$ or more donor cells in whole-blood samples [13]. Disease relapse was defined on the basis of morphology, detection of the BCR-ABL fusion gene, or evidence of leukemic cells in either the $\mathrm{BM}$ or other extramedullary organs. Leukemia-free survival (LFS) was defined as the shortest interval between HSCT and relapse or non-relapse mortality (NRM) or the last followup. Transplantation-related toxicity (TRT) was evaluated using standard criteria established by the National Cancer Institute (NCIC; www.ecog.org/general/ctc). Damage to the patients' organs that was due to either GVHD or infectious complications was excluded from this analysis.

\section{Statistical analysis}

Overall survival (OS) and LFS were estimated using the Kaplan-Meier method. GVHD, disease relapse, and NRM rates were estimated using cumulative incidence analysis. For GVHD, death without an event was a competing risk. NRM and relapse were considered to be mutually competing risks. Univariate analysis was conducted using Cox regression. Multivariate analysis was performed using the Cox proportional regression model. SPSS version 16.0 statistical software was used for Kaplan-Meier, univariate, and multiple regression analyses. $\mathrm{R}$ software version 2.15 .2 was used for competing risk analysis for GVHD, NRM, and relapse.

\section{Results}

\section{Characteristics of patients and donors}

The demographic characteristics and relevant transplantation data for the patients who were included in this study are shown in Table 1. The characteristics of the patients who received either HLA-matched HSCT or haploidentical HSCT were not significantly different. Many of the patients achieved $\mathrm{CR}$ and were BCR-ABL fusion gene-negative after several cycles of chemotherapy because all of the patients received imatinib in combination with standard chemotherapy prior to transplantation. In the HLA-matched HSCT group, seven patients were BCR-ABL fusion genepositive, and the $\mathrm{BCR}-\mathrm{ABL}$ gene expression rate before HSCT was $2.95 \%$. In the haploidentical HSCT group, ten patients were $\mathrm{BCR}-\mathrm{ABL}$ fusion gene-positive, and the $\mathrm{BCR}-\mathrm{ABL}$ gene expression rate before HSCT was $2.42 \%$. All 17 patients were BCR-ABL fusion genenegative posttransplantation.

\section{Engraftment and chimerism}

The numbers of MNCs and CD34+ cells are shown in Table 2. Excluding the two patients who received HLAmatched HSCT and the three patients who received haploidentical HSCT who died prior to engraftment, 77 patients achieved engraftment of both neutrophils and platelets. The median time to achieve an absolute neutrophil count (ANC) $>0.5 \times 10^{9}$ cells/L were 14 days (range $9-54$ days) in the patients who received HLA-matched HSCT and 15 days (range 10-22 days) in those who received haploidentical HSCT $(p=0.225)$. The median time until platelet engraftment totaled $>20 \times 10^{9}$ cells/L were 14 days (range 9-210 days) in the patients who received HLAmatched HSCT and 15 days (range 10-33 days) in those who received haploidentical HSCT $(p=0.134)$. All of the surviving patients had complete donor chimerism by 60 days after transplantation.

\section{GVHD and toxicity}

The cumulative incidence of acute GVHD (aGVHD) among the patients who received haploidentical HSCT (24 patients, $51.1 \%$; $95 \%$ (CI), 35.8-64.4\%) was significantly higher than that among those who received HLA-matched HSCT (9 patients, $25.7 \%$; $95 \%$ (CI), $12.6-41.1 \%)(p=0.026$, Fig. 1a). There was no difference in the incidence of grades III-IV aGVHD between the patients who received HLA-matched HSCT (11.4 \%; $95 \%$ (CI), 3.5-24.5\%) and those who received haploidentical HSCT (17.0\%; 95 \% (CI), 7.8-29.1\%) $(p=0.504$, Fig. $1 \mathrm{~b})$. The 2-year cumulative incidence of chronic GVHD (cGVHD) was higher among the patients who received haploidentical HSCT (23 patients, $48.9 \%, 95 \%$ (CI), 33.8-62.4 \%) than among those who received HLA-matched HSCT (9 patients, $25.7 \%$, $95 \%$ (CI), $12.6-41.1 \%$ ) ( $p=0.033$, Fig. 1c). At the time of analysis, four of the patients who received HLA-matched HSCT showed signs of extensive cGVHD (cumulative incidence, $11.4 \%$; $95 \%$ (CI), 3.5-24.5\%), whereas ten of the patients who received haploidentical HSCT showed such signs (cumulative incidence, $21.3 \%$; 95 \% (CI), 10.9-34.0 \%) $(p=0.222$, Fig. 1d).

The toxicities of the regimens are summarized in Table 3. There was no hepatic venous occlusive disease 
Table 1 Characteristics of patients and donors

\begin{tabular}{|c|c|c|c|}
\hline Characteristic & $\begin{array}{l}\text { HLA-matched HSCT } \\
(n=35)\end{array}$ & $\begin{array}{l}\text { Haploidentical HSCT } \\
(n=47)\end{array}$ & $P$ \\
\hline Age, years, median (range) & $35(5-52)$ & $35(8-50)$ & 0.679 \\
\hline Males/females, $n$ & $22 / 13$ & $24 / 23$ & 0.287 \\
\hline WBC count at diagnose $\times 10^{9}$, median (range) & 45.0(1.4-199.2) & $36.3(1.6-274.4)$ & 0.147 \\
\hline \multicolumn{4}{|l|}{ CNS involvement } \\
\hline Yes/no & $3 / 32$ & $6 / 41$ & 0.548 \\
\hline \multicolumn{4}{|l|}{ Disease status at transplantation } \\
\hline CR1 & 26 & 37 & 0.584 \\
\hline$>C R 1$ & 9 & 10 & \\
\hline \multicolumn{4}{|l|}{ BCR-ABL at transplantation } \\
\hline Negative/positive & $28 / 7$ & $37 / 10$ & 0.888 \\
\hline Average mos. from diagnosis to transplant (range) & $9.0(3-47)$ & $7.0(2-19)$ & 0.104 \\
\hline Cycles of prior chemotherapy (range) & $5.3(2-22)$ & $4.6(2-13)$ & 0.411 \\
\hline ABO match, no. (\%) & & & 0.224 \\
\hline Matched & 12 & 27 & \\
\hline Minor mismatched & 8 & 7 & \\
\hline Major mismatched & 11 & 9 & \\
\hline Major and minor mismatched & 4 & 4 & \\
\hline Donor-recipient sex match & & & 0.323 \\
\hline Male-male & 10 & 16 & \\
\hline Male-female & 9 & 18 & \\
\hline Female-male & 12 & 8 & \\
\hline Female-female & 4 & 5 & \\
\hline
\end{tabular}

(HVOD) in either group. The incidences of creatinine elevation, hemorrhagic cystitis, and heart dysfunction were very low in both groups. The incidence of CMV infection was significantly higher among the patients who received haploidentical HSCT than among those who received HLA-matched HSCT. Among the patients who received haploidentical HSCT, three developed CMV pneumonia and one developed CMV enteritis. Two of these patients ultimately died of CMV pneumonia. The incidences of other toxicities were not significantly different between the two groups.

Table 2 Cell yield (cells/kg recipient body weight) given in average values with minimum and maximum in parentheses

\begin{tabular}{lcc}
\hline & $\mathrm{MNC}\left(\times 10^{8}\right)$ & $\mathrm{CD} 34+\left(\times 10^{6}\right)$ \\
\hline G-PBSCs graft & & \\
For matched HSCT & $9.48(4.27-20.5)$ & $6.48(1.39-31.60)$ \\
G-PBSCs graft & & \\
$\quad$ For haploidentical HSCT & $7.55(2.5-19.71)$ & $4.92(1.03-12.71)$ \\
G-BM & & \\
$\quad$ For haploidentical HSCT & $4.41(1.6-8.46)$ & $1.56(0.54-3.64)$ \\
\hline
\end{tabular}

Relapse, NRM, OS, and LFS

Five of the transplant patients died before engraftment was achieved, and 77 achieved molecular CR after transplantation. The patients who received HLA-matched HSCT were monitored for a median time of 26 months (range 0.2-78 months), and those who received haploidentical HSCT were monitored for a median time of 31 months (range 0-81 months). Relapse was defined as molecular relapse, hematological relapse, or extramedullary leukemia relapse. The incidence of relapse was higher among the patients who received HLA-matched HSCT (14 patients) than among those who received haploidentical HSCT (9 patients) (cumulative incidence, 44.8 vs. $19.1 \%$; $95 \%$ (CI), $23.8-63.7 \%$ vs. $9.3-31.6 \%)(p=0.036$, Fig. 2a). The median time until relapse was 4.6 months in the patients who received HLA-matched HSCT and 7.0 months in those who received haploidentical HSCT. After recurrence, three patients in the HLA-matched HSCT group and two in the haploidentical HSCT group ceased treatment and died a few weeks later. The other patients who experienced relapse received imatinib treatment, and several of them also received chemotherapy, intrathecal chemotherapy, DLI, or biotherapy. The remaining six patients 

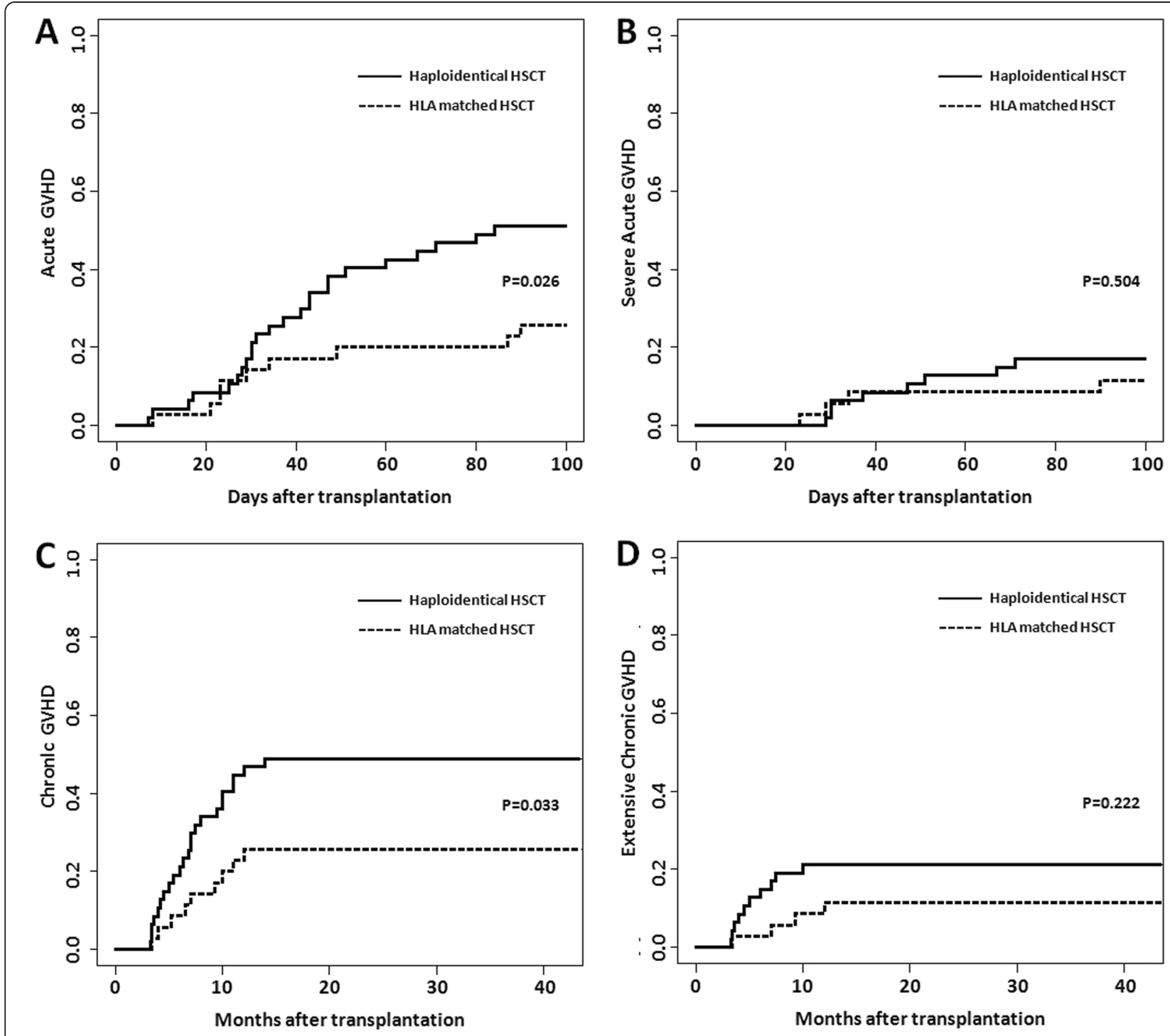

Fig. 1 Cumulative incidence of GVHD. a Acute GVHD, b severe acute GVHD, c chronic GVHD, and d extensive chronic GVHD

in the HLA-matched HSCT group and two in the haploidentical HSCT group are still alive; three of them have central nervous system leukemia (CNSL), and five are BCR-ABL fusion gene-positive. Haploidentical HSCT was associated with a significantly lower risk of relapse, as determined by univariate analysis (Table 4) and confirmed by multivariate analysis (hazard ratio (HR) 0.413 ; $95 \%(\mathrm{CI}), 0.178-0.958)(p=0.039)$. However, $>$ CR1 was a risk factor for recurrence, as determined by univariate (Table 4 ) and multivariate analysis (HR 2.694; 95 \% (CI), 1.121-6.475) ( $p=0.027)$. The patients who had cGVHD appeared to have a lower risk of relapse by univariate analysis but not by multivariate analysis. Other factors, such as age, white blood cell (WBC) count at diagnosis, and the presence of aGVHD, did not affect the risk of relapse.
There was no significant difference in the incidence of NRM between the patients who received HLA-matched HSCT (cumulative incidence, six patients, $17.1 \%$; $95 \%$ (CI), 6.8-31.4\%) and those who received haploidentical HSCT (cumulative incidence, ten patients, $21.3 \%$; $95 \%$ (CI), 10.9-34.1\%) ( $p=0.683$, Fig. 2b). The incidences of NRM were 11.8 and $21.5 \%$ in the BCR-ABL-positive and BCR-ABL-negative patients, respectively.

The Kaplan-Meier estimate of the 2-year OS of the patients who received haploidentical HSCT was $63.8 \%$, which was similar to that of the patients who received HLAmatched HSCT (62.6 \%) ( $p=0.743$, Fig. 2c). Univariate analysis revealed that sex, the WBC count, the presence of GVHD, and HLA disparity did not significantly influence the patients' OS (Table 4). At 2 years, the LFS of the patients who received haploidentical HSCT was higher than 
Table 3 Toxicities by HSCT and infection

\begin{tabular}{|c|c|c|c|}
\hline Toxicity & $\begin{array}{l}\text { HLA-matched } \\
\text { HSCT }\end{array}$ & $\begin{array}{l}\text { Haploidentical } \\
\text { HSCT }\end{array}$ & $P$ \\
\hline \multicolumn{4}{|l|}{ Hepatic } \\
\hline Transaminase elevation & $8(22.9 \%)$ & $14(29.8 \%)$ & 0.484 \\
\hline Bilirubin elevation & $3(8.6 \%)$ & $9(19.1 \%)$ & 0.180 \\
\hline \multicolumn{4}{|l|}{ Gastrointestinal tract } \\
\hline Diarrhea & $25(71.4 \%)$ & $33(70.2)$ & 0.905 \\
\hline Nausea and vomiting & $17(48.6 \%)$ & $32(68.1 \%)$ & 0.075 \\
\hline Mucositis & 13(37.1\%) & $21(44.7 \%)$ & 0.493 \\
\hline Alimentary tract hemorrhage & $2(5.7 \%)$ & $6(12.8)$ & 0.287 \\
\hline \multicolumn{4}{|l|}{ Urinary tract/kidney } \\
\hline Creatinine elevation & 0 & $1(2.1)$ & 0.385 \\
\hline Hemorrhagic cystitis & $1(2.9 \%)$ & 1 & 0.832 \\
\hline \multicolumn{4}{|l|}{ Infection } \\
\hline Bacterial infection & $23(65.7 \%)$ & $32(68.1 \%)$ & 0.821 \\
\hline Fungal infection & $4(11.4 \%)$ & $8(17.0 \%)$ & 0.479 \\
\hline CMV infection & $5(14.3 \%)$ & 18(38.3 \%) & 0.017 \\
\hline \multicolumn{4}{|l|}{ Cardiovascular } \\
\hline Heart dysfunction & 1 & 0 & 0.244 \\
\hline
\end{tabular}

CMV cytomegalovirus

that of the patients who received HLA-matched HSCT $(59.5$ vs. $45.7 \%)(p=0.118)$, although this difference was not statistically significant (Fig. 2d). $>$ CR1 at the time of transplantation significantly influenced LFS, as determined by univariate analysis, but this difference was not confirmed by multivariate analysis (HR 1.895, $95 \%$ (CI), 0.955-3.761) $(p=0.068)$. The cumulative 2-year OS rates were 52.9 and $69.2 \%$ in the BCR-ABL-positive and BCR-ABL-negative patients, respectively $(p=0.325)$. At 2 years, the LFS rates were $41.2 \%$ for the BCR-ABL-positive patients and $58.5 \%$ for the BCR-ABL-negative patients $(p=0.229)$. Although there were no significant differences in OS or PFS due to the limited number of patients included in this study, the BCR-ABL-negative patients tended to have worse outcomes.

\section{Discussion}

$\mathrm{Ph}+\mathrm{ALL}$ is an aggressive disease with a poor prognosis. Despite an initial favorable response to treatment, the long-term outcome is unsatisfactory in adults when TKI/chemotherapy combinations are used without alloHSCT. This unfavorable prognosis might justify the use of alternative conditioning options and higher-risk donors, such as mismatched unrelated donors or haploidentical donors or umbilical cord blood-derived cells. It is difficult to assess the balance between a greater risk of treatment-related mortality (TRM) and an increased risk of relapse. In recent years, considerable progress has been made in haploidentical HSCT, and the clinical outcomes as well as the health-related quality of life (HRQoL) of patients receiving this treatment have been reported to be comparable to those of patients receiving HLA-matched HSCT $[14,15]$. Although haploidentical HSCT has become a common treatment in adult patients who lack a HLA-matched donor, data on its efficacy in Ph+ ALL are limited. We conducted multicenter analysis of 82 $\mathrm{Ph}+\mathrm{ALL}$ patients in southwest China undergoing either HLA-matched HSCT or haploidentical HSCT to examine the effect of haploidentical HSCT on the outcome of Ph+ ALL.

In recent studies, historical comparisons of patients from the pre-TKI era have shown that patients who are treated with TKIs plus chemotherapy have significantly improved CR rates and prolonged LFS [16-18]. Moreover, the pretransplantation use of imatinib improves the outcome of allo-HSCT and patients' candidacy of HSCT [19, 20]. Based on these data, there is now no rationale for omitting TKIs from induction treatment; accordingly, all of the patients in our study received imatinib in combination with standard chemotherapy before transplantation.

Certain studies have found that patients who receive haploidentical HSCT experience significantly delayed myeloid and platelet recoveries compared with those receiving HLA-matched HSCT $[14,21]$. However, we found no difference in engraftment between the patients who received haploidentical HSCT and those who underwent HLA-matched HSCT. Differences between our study and these previous studies include the use of PBMCs plus $\mathrm{BM}$, the lack of in vitro $\mathrm{T}$ cell depletion, and the use of greater numbers of MNCs and CD34+ cells in our study. Different studies have obtained different results regarding the prevalence of GVHD. Luo et al. have reported that grades II-IV aGVHD and severe aGVHD are significantly more frequent in patients undergoing haploidentical HSCT compared with those receiving matched sibling donor HSCT but that the incidence of cGVHD is not significantly affected by donor type [14]. In other studies, the incidence of GVHD has been reported to be similar between patients who have received haploidentical HSCT and those who have undergone matched sibling donor HSCT [21]. Our results showed that the incidences of both aGVHD and cGVHD were higher in patients who received haploidentical HSCT compared with those who received HLA-matched HSCT, but there were no differences between the two patient groups with regard to the incidences of severe aGVHD and extensive cGVHD. Modern treatment strategies have reduced complications in patients receiving haploidentical HSCT, but Wang et al. reported that the incidence of CMV viremia increased to $64 \%$ among patients who underwent haploidentical HSCT [22]. In our study, the incidence of CMV viremia in patients who received haploidentical HSCT was higher than that in those receiving HLA-matched HSCT. This discrepancy 

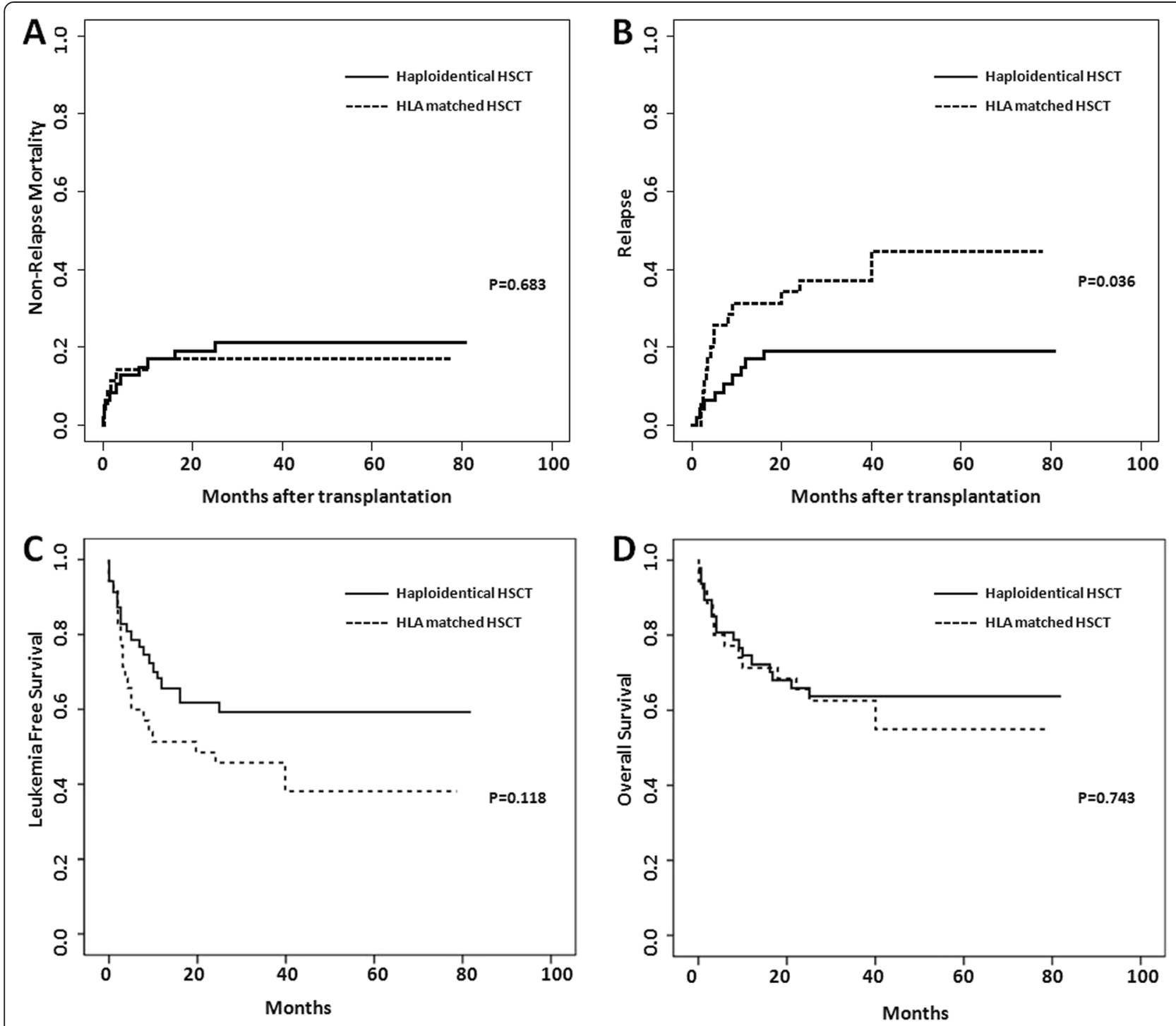

Fig. 2 a Cumulative incidence of NRM. b Cumulative incidence of relapse. c Kaplan-Meier estimate of LFS. d Kaplan-Meier estimate of OS

was associated with the intensified GVHD prophylactic strategy used in haploidentical HSCT [23]. Apart from the incidence of CMV infection, there were no differences in the toxicities of the regimens between the two groups, and no patients in either group experienced HVOD.

The most important finding of this study is that the patients who received haploidentical HSCT had a significantly lower incidence of relapse than those who received HLAmatched HSCT. The lower incidence of relapse among the patients who received haploidentical HSCT may have been due to the graft-versus-leukemia (GVL) activity of haploidentical HSCT. We observed a significantly lower rate of relapse in the patients with cGVHD compared with those without this disease, in agreement with previously published observations [10, 24, 25]. cGVHD has been associated with a putative GVL effect, and several studies have shown that it contributes significantly to the eradication of MRD [10, 24, 25]. The conditioning regimen of haploidentical HSCT is more intensive than that of HLA-matched HSCT, which is related to the lower rate of relapse in haploidentical HSCT. Previous studies have confirmed a relationship between chemotherapeutic regimen intensity and disease recurrence risk. Bredeson et al. [26] compared patients received $\mathrm{FB}$ with those received $\mathrm{BuCy}$, finding that the relapse rate was higher after FB treatment than after $\mathrm{BuCy}$ treatment. Bachanova et al. [27] have confirmed that a less intense regimen may not be sufficient to eliminate residual detectable leukemia.

In our study, the relapse rates were $19.1 \%$ in the patients who received haploidentical HSCT and $44.8 \%$ in those who received HLA-matched HSCT. The relapse rate of the patients who received HLA-matched HSCT 
Table 4 Results of univariate analysis of RE, LFS and OS

\begin{tabular}{|c|c|c|c|c|c|c|}
\hline \multirow[b]{2}{*}{ Variable } & \multicolumn{2}{|l|}{$\mathrm{RE}$} & \multicolumn{2}{|l|}{$\underline{\text { LFS }}$} & \multicolumn{2}{|l|}{ OS } \\
\hline & $\mathrm{HR}(95 \% \mathrm{Cl})$ & $P$ & $\mathrm{HR}(95 \% \mathrm{Cl})$ & $P$ & $\mathrm{HR}(95 \% \mathrm{Cl})$ & $P$ \\
\hline \multicolumn{7}{|l|}{ Age } \\
\hline$<40$ years & 1 (Reference) & & 1 (Reference) & & 1 (Reference) & \\
\hline$\geq 40$ years & $0.752(0.279-2.026)$ & 0.573 & $0.932(0.454-1.913)$ & 0.849 & $0.954(0.427-2.314)$ & 0.910 \\
\hline \multicolumn{7}{|l|}{ HLA disparity } \\
\hline Identical HSCT & 1 (Reference) & & 1 (Reference) & & 1(Reference) & \\
\hline Haploidentical HSCT & 0.399(0.173-0.924) & 0.032 & $0.609(0.325-1.143)$ & 0.123 & 0.889(0.438-1.803) & 0.744 \\
\hline \multicolumn{7}{|c|}{ Duration from diagnosis to SCT } \\
\hline$<180$ days & 1 (Reference) & & 1 (Reference) & & 1 (Reference) & \\
\hline$\geq 180$ days & 1.775(0.783-4.025) & 0.169 & $1.389(0.740-2.609)$ & 0.306 & 0.915(0.444-1.886) & 0.810 \\
\hline \multicolumn{7}{|c|}{ Disease status before transplantation } \\
\hline CR1 & 1 (Reference) & & 1(Reference) & & 1(Reference) & \\
\hline$>C R 1$ & $3.289(1.429-7.569)$ & 0.005 & $2.279(1.179-4.408)$ & 0.014 & $1.711(0.804-3.642)$ & 0.164 \\
\hline \multicolumn{7}{|l|}{ WBC at diagnosis } \\
\hline$<30 \times 10^{9} / \mathrm{L}$ & 1(Reference) & & 1(Reference) & & 1(Reference) & \\
\hline$\geq 30 \times 10^{9} / \mathrm{L}$ & $2.272(1-5.165)$ & 0.05 & $1.842(0.976-3.478)$ & 0.06 & $1.590(0.778-3.249)$ & 0.204 \\
\hline \multicolumn{7}{|l|}{ Acute GVHD } \\
\hline Yes & 1 (Reference) & & 1 (Reference) & & 1 (Reference) & \\
\hline No & $0.562(0.235-1.366)$ & 0.204 & $0.814(0.427-1.552)$ & 0.532 & $1.208(0.595-2.451)$ & 0.601 \\
\hline \multicolumn{7}{|l|}{ Chronic GVHD } \\
\hline Yes & 1 (Reference) & & 1 (Reference) & & 1(Reference) & \\
\hline No & $0.5(0.253-0.988)$ & 0.046 & $0.570(0.241-1.346)$ & 0.2 & $0.584(0.275-1.242)$ & 0.162 \\
\hline \multicolumn{7}{|l|}{ CMV } \\
\hline Positive & 1 (Reference) & & 1(Reference) & & 1(Reference) & \\
\hline Negative & $0.511(0.173-1.506)$ & 0.223 & $1.087(0.55-2.148)$ & 0.81 & $1.508(0.721-3.155)$ & 1.508 \\
\hline
\end{tabular}

appeared to be higher in our study than in previously published reports $[5,18,28]$. This difference might be due to one or a combination of the following factors. First, more patients transplanted in $>C R 1$ were included in our study compared with other studies [5, 28]. Our results showed that $>$ CR1 at transplantation was associated with an increased risk of relapse. Chen et al. also suggested that the remission status at the time of HSCT is significantly predictive of both LFS and OS [5]. Allo-HSCT in CR1 remains the standard of care at most centers [28]. Second, we included not only hematological and extramedullary relapse but also molecular relapse in our study. The patients in our study also did not receive "upfront" imatinib postallogeneic HSCT, and imatinib treatment was only initiated if the BCR-ABL fusion product or extramedullary relapse was detected. Pfeifer et al. observed molecular recurrence in $56 \%$ of patients overall [29]. There is insufficient evidence to date indicating that imatinib should be given to all patients following allogeneic HSCT [5]. Certain centers have reported that imatinib is poorly tolerated following myeloablative allogeneic HSCT [5, 17, 30]. A small study conducted at the University of Minnesota [20] showed a trend toward an improved outcome in patients treated with imatinib during the pre- and posttransplantation periods. Administration of imatinib maintenance therapy after HSCT has also been suggested to reduce the relapse rate and improve LFS in Ph+ ALL patients [5]. The only randomized study of the use of TKIs after allogeneic HSCT is currently being conducted by the GMALL group [29]. This study showed that prophylactic imatinib treatment significantly reduced the incidence of molecular relapse after allogeneic HSCT but that durable PCR negativity could still be achieved in the majority of posttransplantation patients with molecular relapse by restarting imatinib therapy. OS was found to significantly differ between the two treatment arms. Although haploidentical HSCT reduces the risk of disease recurrence, our study found no difference in either LFS or OS, possibly due to the small number of included patients and the beneficial effect of restarting imatinib in most patients.

In conclusion, we found a significant reduction in the relapse rate in $\mathrm{Ph}+\mathrm{ALL}$ patients who have received 
haploidentical HSCT compared with patients who have undergone HLA-matched HSCT. The incidences of aGVHD, cGVHD, and CMV viremia were higher in the patients who received haploidentical HSCT than in those who received HLA-matched HSCT, but there was no difference in NRM between these two groups. Although a well-designed, larger prospective study is needed to define the role of haploidentical HSCT in disease progression in patients with $\mathrm{Ph}+\mathrm{ALL}$, our results suggest that haploidentical HSCT is a promising option for $\mathrm{Ph}+\mathrm{ALL}$ patients who lack a suitable HLA-matched donor.

\begin{abstract}
Abbreviations
aGVHD: acute graft-versus-host disease; allo-HSCT: allogeneic hematopoietic stem cell transplantation; ANC: absolute neutrophil count;

Ara-C: arabinosylcytosine; ATG: anti-thymocyte globulin; BM: bone marrow; Bu: busulfan; CGVHD: chronic graft-versus-host disease;

CMV: cytomegalovirus; CR: complete remission; CR1: first complete remission; CSA: cyclosporin; CY: cyclophosphamide; FB: fludarabine and busulfan; FK506: tacrolimus; G-BM: mobilize stem cells in the bone marrow;

G-CSF: granulocyte colony stimulating factor; G-PB: mobilize stem cells in the peripheral blood; GVHD: graft-versus-host disease; GVL: graft-versus-leukemia; HRQoL: health-related quality of life; HSCT: hematopoietic stem cell transplantation; HVOD: hepatic venous occlusive disease; LFS: leukemia-free survival; MMF: mycophenolate mofetil; MNCs: mononuclear cells; MTX: methotrexate; NRM: non-relapse mortality; OS: overall survival; PB: peripheral blood; PBMC: peripheral blood mononuclear cell; Ph+ ALL: Philadelphia chromosome-positive acute lymphoblastic leukemia; QRT-PCR: real-time quantitative reverse transcription polymerase chain reaction; TBI: total-body irradiation; TKI: tyrosine kinase inhibitors; TRT: transplantation-related toxicity; WBC: white blood cell.
\end{abstract}

\section{Competing interest}

The authors declare that they have no competing interests.

\section{Authors' contributions}

All authors have contributed to data preparation, drafting, and revising the manuscripts. All authors have read and approved the final manuscript.

\section{Acknowledgements}

This work was supported by the Chinese National Natural Science Foundation (No. 81270569, No 81070388), the Natural Science Foundation Project of CQ CSTC(cstc2012jjA10049), Research Foundation of Janssen Research Council China(JRCC2011Tumor01).

\section{Author details}

${ }^{1}$ Department of Hematology, Xinqiao Hospital, Third Military Medical University, Xinqiao Street, Shangpinba District, Chongqing 400037, China. ${ }^{2}$ Department of Hematology, General Hospital of Chengdu Military Region of PLA, Sichuan, China. ${ }^{3}$ Department of Hematology, General Hospital of Kunming Military Region of PLA, Yunnan, China. ${ }^{4}$ Department of Hematology, Yunnan Provincial Peoples Hospital, Yunnan, China. ${ }^{5}$ Department of Hematology, Second Yunnan Provincial Peoples Hospital, Yunnan, China. ${ }^{6}$ Department of Hematology, The Affiliated Hospital of Zunyi Medical College, Zunyi, Guizhou, China.

Received: 8 April 2015 Accepted: 8 July 2015

Published online: 26 July 2015

\section{References}

1. Moorman AV, Harrison CJ, Buck GA, Richards SM, Secker-Walker LM, Martineau $M$, et al. Karyotype is an independent prognostic factor in adult acute lymphoblastic leukemia (ALL): analysis of cytogenetic data from patients treated on the Medical Research Council (MRC) UKALLXII/Eastern Cooperative Oncology Group (ECOG)2993 trial. Blood. 2007;109(8):3189-97.

2. Yanmin Z, He H, Guoqing W. Novel agents and biomarkers for acute lymphoid leukemia. J Hematol Oncol. 2013;6:40.
3. Ribera JM. Optimal approach to treatment of patients with Philadelphia chromosome-positive acute lymphoblastic leukemia: how to best use all the available tools. Leuk Lymphoma. 2013;54(1):21-7.

4. Rives S, Estella J, Gómez P, López-Duarte M, de Miguel PG, Verdeguer A, et al. Intermediate dose of imatinib in combination with chemotherapy followed by allogeneic stem cell transplantation improves early outcome in paediatric Philadelphia chromosome-positive acute lymphoblastic leukaemia (ALL): results of the Spanish Cooperative Group SHOP studies ALL-94, ALL-99 and ALL-2005. Br J Haematol. 2011;154(5):600-11.

5. Chen H, Liu KY, Xu LP, Liu DH, Chen YH, Zhao XY, et al. Administration of imatinib after allogeneic hematopoietic stem cell transplantation may improve disease-free survival for patients with Philadelphia chromosome-positive acute lymphoblastic leukemia. J Hematol Oncol. 2012;5:29.

6. de Labarthe A, Rousselot P, Huguet-Rigal F, Delabesse E, Witz F, Maury S, et al. Imatinib combined with induction or consolidation chemotherapy in patients with de novo Philadelphia chromosome-positive acute lymphoblastic leukemia: results of the GRAAPH-2003 study. Blood. 2007;109(4):1408-13.

7. Fielding AK, Rowe JM, Richards SM, Buck G, Moorman AV, Durrant IJ, et al. Prospective outcome data on 267 unselected adult patients with Philadelphia chromosome-positive acute lymphoblastic leukemia confirms superiority of allogeneic transplantation over chemotherapy on the pre-imatinib era: results from the international ALL trial MRC UKALLXII/ ECOG2993. Blood. 2009;113(19):4489-96.

8. Onishi Y, Sasaki O, Ichikawa S, Inokura K, Katsuoka Y, Ohtsuka Ohba R, et al. Favorable outcome of unrelated cord blood transplantation for Philadelphia chromosome-positive acute lymphoblastic leukemia. Biol Blood Marrow Transplant. 2011;17(7):1093-7.

9. Chen XH, Gao L, Zhang X, Gao L, Zhang C, Kong PY, et al. HLAhaploidentical blood and bone marrow transplantation with anti-thymocyte globulin: long-term comparison with HLA-identical sibling transplantation. Blood Cells Mol Dis. 2009:43(1):98-104.

10. Mo XD, Xu LP, Zhang XH, Liu DH, Wang Y, Chen $\mathrm{H}$ et al. Haploidentical hematopoietic stem cell transplantation in adults with Philadelphia-negative acute lymphoblastic leukemia: no difference in the high- and low-risk groups. Int J Cancer 2014; epub ahead of print 20 Aug 2014; doi: 10.1002/ijc.29146.

11. Raiola AM, Dominietto A, di Grazia C, Lamparelli T, Gualandi F, Ibatici A, et al. Unmanipulated haploidentical transplants compared with other alternative donors and matched sibling grafts. Biol Blood Marrow Transplant. 2014;20(10):1573-9.

12. Yong-rong L, Yu-hong C, Deng-ming H, Ming J, Qi-fa L, Lin L, et al. Multicenter phase ii study of a combination of cyclosporine a, methotrexate and mycophenolate mofetil for GVHD prophylaxis: results of the Chinese Bone Marrow Transplant Cooperative Group (CBMTCG). J Hematol Oncol. 2014;7:59.

13. Hui L, Xiao Z, Zhaoyang S, Jing S, Yang X, Danian N, et al. Busulfan plus fludarabine as a myeloablative conditioning regimen compared with busulfan plus cyclophosphamide for acute myeloid leukemia in first complete remission undergoing allogeneic hematopoietic stem cell transplantation: a prospective and multicenter study. J Hematol Oncol. 2013;6:15.

14. Luo Y, Xiao H, Lai X, Shi J, Tan Y, He J, et al. T-cell-replete haploidentical HSCT with low-dose anti-T-lymphocyte globulin compared with matched sibling HSCT and unrelated HSCT. Blood. 2014;124(17):2735-43.

15. Mo XD, Xu LP, Liu DH, Chen YH, Han W, Zhang XH, et al. Patients receiving HLA-haploidentical/partially matched related allo-HSCT can achieve desirable health-related QoL that is comparable to that of patients receiving HLAidentical sibling allo-HSCT. Bone Marrow Transplantation. 2012;47(9):1201-5.

16. Bassan R, Rossi G, Pogliani EM, Di Bona E, Angelucci E, Cavattoni I, et al. Chemotherapy-phased imatinib pulses improve long-term outcome of adult patients with Philadelphia chromosome-positive acute lymphoblastic leukemia: Northern Italy Leukemia Group protocol 09/00. J Clin Oncol. 2010;28(22):3644-52.

17. Ribera JM, Oriol A, González M, Vidriales B, Brunet S, Esteve J, et al. Concurrent intensive chemotherapy and imatinib before and after stem cell transplantation in newly diagnosed Philadelphia chromosome-positive acute lymphoblastic leukemia: final results of the CSTIBESO2 trial. Haematologica. 2010;95(1):87-95.

18. Mizuta S, Matsuo K, Yagasaki F, Yujiri T, Hatta Y, Kimura Y, et al. Pre-transplant imatinib-based therapy improves the outcome of allogeneic hematopoietic stem cell transplantation for BCR-ABL-positive acute lymphoblastic leukemia. Leukemia. 2011;25(1):41-7.

19. Towatari M, Yanada M, Usui N, Takeuchi J, Sugiura I, Takeuchi M, et al. Combination of intensive chemotherapy and imatinib can rapidly induce high-quality complete remission for a majority of patients with newly 
diagnosed BCR-ABL-positive acute lymphoblastic leukemia. Blood. 2004;104(12):3507-12.

20. Burke MJ, Trotz B, Luo X, Baker KS, Weisdorf DJ, Wagner JE, et al. Allohematopoietic cell transplantation for Ph chromosome-positive ALL: impact of imatinib on relapse and survival. Bone Marrow Transplant. 2009;43(2):107-13.

21. Di Stasi A, Milton DR, Poon LM, Hamdi A, Rondon G, Chen J, et al. Similar transplantation outcomes for acute myeloid leukemia and myelodysplastic syndrome patients with haploidentical versus 10/10 human leukocyte antigen matched unrelated and related donors. Biol Blood Marrow Transplant. 2014;20(12):1975-81.

22. Wang Y, Liu DH, Liu KY, Xu LP, Zhang XH, Han W, et al. Long-term follow-up of haploidentical hematopoietic stem cell transplantation without in vitro T cell depletion for the treatment of leukemia. Cancer. 2013;119(5):978-85.

23. Ren L, Qifa L. Diagnosis and treatment of viral diseases in recipients of allogeneic hematopoietic stem cell transplantation. J Hematol Oncol. 2013;6:94.

24. Chim CS, Lie AK, Liang R, Au WY, Kwong YL. Long-term results of allogeneic bone marrow transplantation for 108 adult patients with acute lymphoblastic leukemia: favorable outcome with BMT at first remission and HLA-matched unrelated donor. Bone Marrow Transplant. 2007;40(4):339-47.

25. Lee S, Chung NG, Cho BS, Eom KS, Kim YJ, Kim HJ, et al. Donor-specific differences in long-term outcomes of myeloablative transplantation in adults with Philadelphia-negative acute lymphoblastic leukemia. Leukemia. 2010;24(12):2110-9.

26. Bredeson CN, Zhang MJ, Agovi MA, Bacigalupo A, Bahlis NJ, Ballen K, et al. Outcomes following HSCT using fludarabine, busulfan, and thymoglobulin: a matched comparison to allogeneic transplants conditioned with busulfan and cyclophosphamide. Biol Blood Marrow Transplant. 2008;14(9):993-1003.

27. Bachanova V, Marks DI, Zhang MJ, Wang H, de Lima M, Aljurf MD, et al. Ph+ ALL patients in first complete remission have similar survival after reduced intensity and myeloablative allogeneic transplantation: impact of tyrosine kinase inhibitor and minimal residual disease. Leukemia. 2014;28(3):658-65

28. Thyagu S, Minden MD, Gupta V, Yee KW, Schimmer AD, Schuh AC, et al. Treatment of Philadelphia chromosome-positive acute lymphoblastic leukaemia with imatinib combined with a paediatric-based protocol. $\mathrm{Br}$ Haematol. 2012;158(4):506-14.

29. Pfeifer H, Wassmann B, Bethge W, Dengler J, Bornhäuser M, Stadler M, et al. Randomized comparison of prophylactic and minimal residual diseasetriggered imatinib after allogeneic stem cell transplantation for BCR-ABL1positive acute lymphoblastic leukemia. Leukemia. 2013;27(6):1254-62.

30. Anderlini P, Sheth S, Hicks K, Ippoliti C, Giralt S, Champlin RE.

Re:Imatinibmesylate administration in the first 100 day after stem cell transplantation [letter]. Biol Blood Marrow Transplant. 2004;10(12):883-4.

\section{Submit your next manuscript to BioMed Central and take full advantage of:}

- Convenient online submission

- Thorough peer review

- No space constraints or color figure charges

- Immediate publication on acceptance

- Inclusion in PubMed, CAS, Scopus and Google Scholar

- Research which is freely available for redistribution

Submit your manuscript at www.biomedcentral.com/submit 